\title{
Silicone Oil Droplets in Vitreous after Intravitreal Bevacizumab Injection
}

\author{
Haroon Tayyab, Asad Aslam Khan, Muhammad Ali A Sadiq, Sana Jahangir, Suhail Sarwar
}

Pak J Ophthalmol 2018, Vol. 35, No. 2

See end of article for authors affiliations

Correspondence to:

Dr. Haroon Tayyab

Assistant Prof. of Vitreoretina

COAVS - KEMU (Mayo Hospital

Lahore)

Email:

haroontayyab79@gmail.com

\begin{abstract}
Intravitreal injection of Bevacizumab for various retinal vascular disorders has become the mainstay of treatment. The injection of Bevacizumab is usually dispensed through local pharmacies after formulating from a larger vial under sterile conditions. We report an interesting finding of silicone oil bubble in vitreal cavity after intravitreal Bevacizumab injection. This is attributed to the fact that the syringes used for dispensing bevacizumab are coated with silicone oil that acts as a lubricant. These silicone oil droplets can lead to floaters and other possible complications. We report two cases of such occurrence and propose strategies to avoid this complication.
\end{abstract}

Keywords: Bevacizumab, diabetic macular edema, silicone oil, ranibizumab.
$\mathrm{I}$ n last one decade, intravitreal drug delivery in the form of anti-vascular endothelial growth factor (anti-VEGF) has become the mainstay of treatment for many retinal vascular disorders including diabetic retinopathy, diabetic maculopathy and macular edema resulting from retinal vein occlusions ${ }^{1,2}$. Also, the current recommended treatment for wet age related macular degeneration (ARMD) is intravitreal antiVEGF injections ${ }^{3}$. Given the highly prevalent nature of conditions like diabetic maculopathy and wet ARMD, it is no surprise to us that intravitreal injections of bevacizumab, ranibizumab and aflibercept have become the most commonly performed vitreoretinal intervention and second most commonly performed ophthalmic surgical procedure. There have been many studies where local and systemic side effects of intravitreal injections have been mentioned. ${ }^{4}$ An interesting finding after injection of bevacizumab and ranibizumab is finding of silicone oil bubble in vitreous cavity. This can be symptomatic in the form of a floater or an incidental finding during detailed retinal examination. First of such reports was by Fruend and Richard F Spaide in 20065. This peculiar finding has been infrequently but persistently reported in western literature up till mid $2016^{6}$. This finding has been attributed to the fact that intravitreal 
injection syringes are pretreated with silicone oil that acts as a lubricating agent for smooth entry in the tissue ${ }^{7}$.

Here we report our first few cases of intravitreal silicone oil bubble in patients who had received Bevacizumab for diabetic macular edema. To date, local literature has provided no such report about this interesting finding. The rationale of reporting these cases is to discuss possible ocular implications and propose remedy to this problem.

These case reports are from the outdoor patient department (OPD) of Ophthalmology Unit 3, Mayo hospital, Lahore from the period between October 2017 to February 2018. An informed consent was sought from both patients and hospital ethics committee approval was obtained before proceeding with reporting of these cases. While presenting these case series, the authors do not have any conflict of interest to disclose.

\section{Case 1}

Our first case (October 2017) was a 54 year old male diabetic patient who had been on intravitreal Bevacizumab injection for his diabetic macular edema in left eye. He had history of 7 such injections in his right eye and presented to our outdoor patient department for routine follow-up. His last injection was one week ago. He had no complaints of floaters at the time of presentation. His best corrected visual acuity in left eye was 20/80 and intraocular pressure was $15 \mathrm{mmHg}$. He had a history of uneventful phacoemulsification with IOL implantion one year ago. A fundus photograph was taken using Topcon Fundus camera (TRC-50DX Topcon Medical Systems, Inc. Oakland, NJ. USA). The next follow-up of this patient was in December 2017 by which time the silicone oil bubble was no more visible on slit lamp examination. This patient has remained complication free and symptom free to date as far as the silicone oil bubble was concerned (Fig. 1). B-scan was performed and classic ringing bell sign due to silicone oil droplet was noted (Fig. 2). The size of silicone oil droplet was noted to be $0.3 \mathrm{~mm}$.

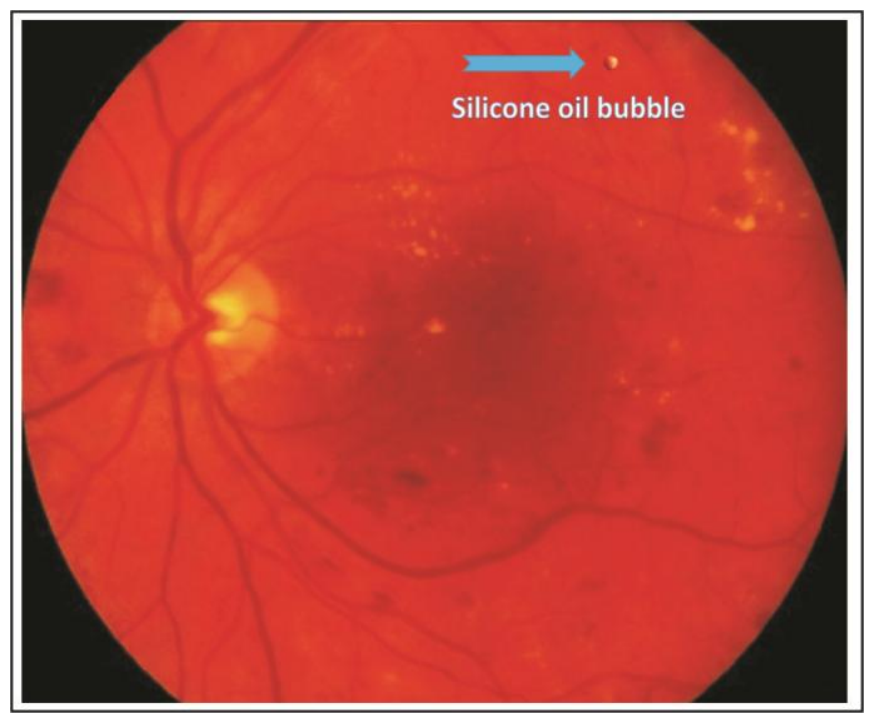

Fig. 1: Silicone oil bubble in vitreous cavity after intravitreal Bevacizumab injection - Left eye.

\section{Case 2}

The second case (February 2018) was a 60 year old female who presented to OPD and was scheduled to have her left eye examination prior to intravitreal Bevacizumab injection for diabetic macular edema. This was her third injection in left eye. Her best corrected visual acuity was 20/200 and intraocular pressure was $17 \mathrm{~mm} \mathrm{Hg}$. She was phakic with no 
history of ocular surgery apart from intravitreal Bevacizumab. We noted intravitreal silicone oil bubble suspended in superior half of post vitreous cavity but could not capture this finding on fundus camera due to very small and indistinct appearance of this bubble on photography. Her last injection was one month ago. She also remained symptom free.

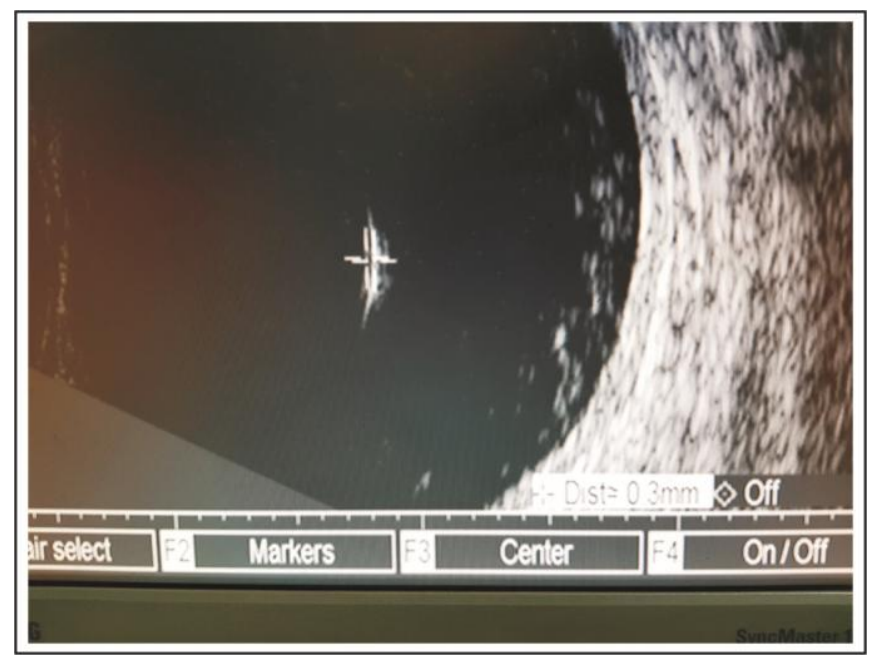

Fig. 2: Ringing bell sign on B - scan. Size of silicone oil bubble is $0.3 \mathrm{~mm}$.

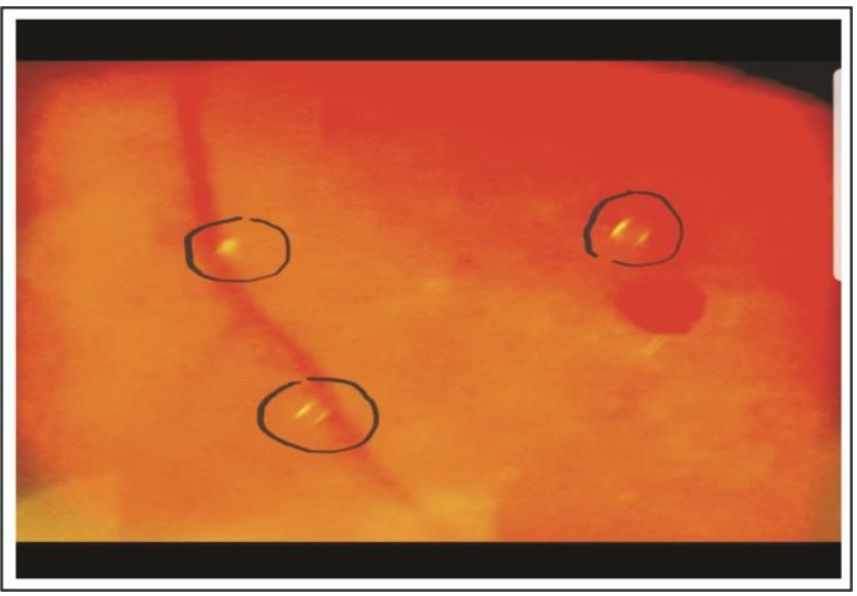

Fig. 3: Three distinct silicone oil bubbles in vitreous cavity (marked in black circle).

\section{Case 3:}

Our third case was a 60 year old lady who presented to us with traumatic crystalline dislocation after blunt trauma in her right eye. On detailed fundus examination, she was found to have old central retinal vein occlusion with subhyaloid hemorrhage for which she had received 2 bevacizumab injections 8 months ago. She was also noted to have 2 silicone oil bubbles on her vitreous cavity. Her fundus photograph shows silicone oil bubbles suspended in vitreous cavity (Fig. 3). She also did not have any history of ocular surgery apart from mentioned intervention for her CRVO.

\section{DISCUSSION}

The most frequent anti-VEGF used in Pakistan is Bevacizumab. It is usually dispensed by pharmacies in pre-formulated insulin syringes from a larger vial under sterile conditions. The largest dispenser of Bevacizumab in the province of Punjab, Pakistan is Shaukat Khanum Memorial Cancer Hospital and Research Centre (SKMCH). We have not noted any clinical report about the presence of silicone oil bubbles after intravitreal Bevacizumab injections in local literature. This problem was recently reported by American Society of Retina Specialists (ASRS) in 2016, where they attributed this finding to silicone oil lining the syringe and needle that acts as a lubricating agent to facilitate movement ${ }^{6}$.

In our clinical practice, lots of patients report of floaters after intravitreal Bevacizumab injection but it was never attributed to silicone oil droplets. Only recently that we started noting presence of silicone oil bubbles in vitreous cavity after this intervention and thus reported the three cases. None of our patients had any symptoms related to silicone oil bubble because on both occasions the bubble was above and away from the nodal point; and on one occasion the BCVA was decreased to an extent that floater may not have been noted by the patient. In one of the largest case series reported in American Journal of Ophthalmology, it was quoted that the rate of this finding was $0.026 \%$ per injection or $0.15 \%$ per patient (A total of 7 patients reported). ${ }^{6}$ With time the complaints of patient decreased because the oil bubble rose superiorly to pars plicata region of fundus and became un-noticeable to patient and ophthalmologist alike. Polydimethylsiloxane (PDMS) is the most frequently used silicone oil to lubricate the syringes and is density is less than vitreous (PDMS: $965 \mathrm{~kg} / \mathrm{m} 3$; Vitreous $1000 \mathrm{~kg} / \mathrm{m} 3$ ).

It should be remembered that Bevacizumab is dispensed for intravitreal use in insulin syringes that are manufactured with the intent of subcuticular use especially in diabetic patients. We use syringes manufactured by Becton Dickinson (BD) and Company (30 G; 12.7mm length; U100 $1 \mathrm{ml}$ Insulin 
Syringe BD Ultra-Fine ${ }^{\mathrm{TM}}$ needle, Becton Dickinson and Company, Franklin Lakes, NJ). BD states that needles and syringes are lubricated with silicone oil during the manufacturing process to facilitate smooth drug delivery and less leaching of drug with syringe and needle walls. The lubricant most likely used is 365 Dow Corning surfactant that contains 2-3\% of silicone oil 7 . The Dow Corning company has claimed that there are no adverse effects related to its surfactant and that this surfactant has also been used in other products that have human contact including food industry ${ }^{8}$.

Kocabora reported similar finding while using injections of Pegabtanib9 ${ }^{9}$. Pegabtanib was packaged in a sterile, 1-mL, USP type 1, graduated, prefilled glass syringe fitted with a sterile 27-gauge needle manufactured by BD (similar manufacturing process). ${ }^{7}$ Ranibizumab is drawn up with a 19-gauge filter needle (5-um filter) and injected intravitreal using a 30-gauge 0.5-in needle (Precision Glide, Becton Dickinson and Company, Franklin Lakes, NJ).

Since these syringes are very commonly used in diabetic patients, there is compelling evidence of presence of silicone oil in these syringes in diabetic literature. A subcuticular granulomatous reaction induced by silicone oil was found in one case series. ${ }^{10}$ Miller et al documents the extrusion of silicone oil from these syringes after repeated flushing with distilled water. In another report, silicone oil became incorporated in insulin after repeated pumping of plunger in the syringe shaft ${ }^{9}$. The efficacy of insulin has also been reported to be decreased after getting mixed with silicone oil. This area still remains to be unexplored whether the efficacy of anti-VEGFs is reduced after coming in contact with silicone oil. Perhaps further insight into this problem will give us a better idea about the interaction of anti-VEGFs and silicone oil.

We need to review the methodology by which pharmacies dispense Bevacizumab despite no adverse effects have been reported due to the problem of inadvertent silicone oil injection in eye.

Since it has been reported that repeated pumping of plunger can lead to release of silicone oil from hub and syringe lining, it is suggested that the drug should be withdrawn from the vial in the syringe with minimal manipulation of plunger. We also recommend to perform Bevacizumab stability test after being packaged in the syringe to ensure its efficacy and to avoid any potential degradation.
Syringes have been in use which incorporate silicone oil free surfactant as lubricant but they are slightly more expensive ${ }^{6}$. The use of such syringes can also be suggested to avoid this potential problem.

In my impression, the incidence of this finding is more than what we have observed in our recent experience. Possible reasons may include ignorance towards this potential problem, busy outdoor patient department schedules, tiny size and innocuous nature of the droplet and the likelihood of this droplet to rise up and become un-noticeable to the examining ophthalmologist.

\section{CONCLUSION}

In context of this interesting observation, we recommend following changes in the process of dispensing and injecting Bevacizumab:

- Education of pharmacists to prepare intravitreal Bevacizumab with minimal manipulation of plunger.

- Use of Silicon oil polymer free syringes (can be discussed with SKMCH).

- Revision of consent forms for patients where this problem is well explained to the patients.

\section{Author's Affiliation}

Dr. Haroon Tayyab

Assistant Prof. of Vitreoretina - COAVS - KEMU (Mayo Hospital Lahore)

Prof. Dr. Asad Aslam Khan

Professor \& Head Department of Ophthalmology KEMU (Mayo Hospital Lahore)

Dr. Muhammad Ali A Sadiq

Assistant Prof. of Ophthalmology - KEMU (Mayo Hospital Lahore)

Dr. Sana Jahangir

Assistant Prof. of Ophthalmology - Sharif Medical \& Dental College - Lahore

Dr. Suhail Sarwar

Associate Prof. of Diagnostic Opthalmology - COAVSKEMU (Mayo Hospital Lahore) 


\section{Role of Authors}

Dr. Haroon Tayyab

Principle investigator, article write-up.

Prof. Dr. Asad Aslam Khan

Literature search

Dr. Muhammad Ali A Sadiq

Literature search, discussion.

Dr. Sana Jahangir

Photography, data maintenance.

Dr. Suhail Sarwar

B-Scan, photography.

\section{REFERENCES}

1. Miller K, Fortun JA. Diabetic Macular Edema: Current Understanding, Pharmacologic Treatment Options, and Developing Therapies. Asia Pac J Ophthalmol (Phila). 2018 Jan-Feb; 7 (1): 28-35.

2. Brogan K, Precup M, Rodger A, Young D, Gilmour DF. Pre-treatment clinical features in central retinal vein occlusion that predict visual outcome following intravitreal ranibizumab. BMC Ophthalmol. 2018 Feb. 9; 18 (1): 37.

3. Miere A, Oubraham H, Amoroso F, Butori P, Astroz P, Semoun O, et al. Optical Coherence Tomography
Angiography to Distinguish Changes of Choroidal Neovascularization after Anti-VEGF Therapy: Monthly Loading Dose versus Pro Re Nata Regimen. J Ophthalmol. 2018 Feb. 4; 2018: 3751702.

4. Gupta A, Sun JK, Silva PS. Complications of Intravitreous Injections in Patients with Diabetes. Semin Ophthalmol. 2018; 33 (1): 42-50.

5. Freund KB, Laud K, Eandi CM, Spaide RF. Silicone oil droplets following intravitreal injection. Retina. 2006 Jul-Aug; 26 (6): 701-703.

6. Jea H. Yu, Esmeralda Gallemore, Jisoo K. Kim, Rocky Patel, Jorge Calderon, Ron P. Gallemore. Silicone oil droplets following intravitreal bevacizumab injections. American Journal of Ophthalmology Case Reports, 2018; 10: 142-144.

7. Bakri SJ, Ekdawi NS. Intravitreal silicone oil droplets after intravitreal drug injections. Retina. 2008 Jul-Aug; 28 (7): 996-1001.

8. Corporation DC. Corning 365, 35\% Dimethicone NF Emul- sion. Midland, Michigan: Medical Products Division; 2002.

9. Kocabora MS, Ozbilen KT, Serefoglu K. Intravitreal silicone oil droplets following pegaptanib injection. Acta Ophthalmol. 2010 Mar; 88 (2): e44-45.

10. Travis WD, Balogh $\mathbf{K}$, Abraham JL. Silicone granulomas: report of three cases and review of the literature. Hum Pathol. 1985 Jan; 16 (1): 19-27. 